\title{
A brief analysis of self-gravitating polytropic models with a non-zero cosmological constant (Research Note)
}

\author{
M. Merafina ${ }^{1}$, G. S. Bisnovatyi-Kogan ${ }^{2}$, and S. O. Tarasov ${ }^{3}$ \\ ${ }^{1}$ University of Rome "La Sapienza", Department of Physics, Piazzale Aldo Moro 2, 00185 Rome, Italy \\ e-mail: marco.merafina@roma1.infn.it \\ 2 Space Research Institute (IKI) Profsoyuznaya 84/32, 117997 Moscow, Russia \\ e-mail: gkogan@iki.rssi.ru \\ 3 National Research Nuclear University MEPHI, Kashirskoe Shosse 31, 115409 Moscow, Russia \\ e-mail: trsvsrg@mail.ru
}

Received 21 September 2011 / Accepted 24 February 2012

\section{ABSTRACT}

\begin{abstract}
Context. We investigate the equilibrium and stability of polytropic spheres in the presence of a non-zero cosmological constant. Aims. We solve the Newtonian gravitational equilibrium equation for a system with a polytropic equation of state of the matter $P=K \rho^{\gamma}$ introducing a non-zero cosmological constant $\Lambda$.

Methods. We consider the cases of $n=1,1.5,3$ and construct series of solutions with a fixed value of $\Lambda$. For each value of $n$, the non-dimensional equilibrium equation has a family of solutions, instead of the unique solution of the Lane-Emden equation at $\Lambda=0$. Results. The equilibrium state exists only for central densities $\rho_{0}$ higher than the critical value $\rho_{\mathrm{c}}$. There are no static solutions at $\rho_{0}<\rho_{\mathrm{c}}$. We investigate the stability of equilibrium solutions in the presence of a non-zero $\Lambda$ and show that dark energy reduces the dynamic stability of the configuration. We apply our results to the analysis of the properties of the equilibrium states of clusters of galaxies in the present universe with non-zero $\Lambda$.
\end{abstract}

Key words. dark matter - dark energy - galaxies: clusters: general

\section{Introduction}

Detailed analysis of the observations of distant SN Ia (Riess et al. 1998; Perlmutter et al. 1999) and the spectrum of fluctuations in the cosmic microwave background radiation (CMB) (see e.g. Spergel et al. 2003) have lead to the conclusion that the term representing "dark energy" (DE) contains about $70 \%$ of the average energy density in the present universe and its properties are very close (identical) to the properties of the Einstein cosmological $\Lambda$ term. In the papers of Chernin (see review 2008), the question was raised about the possible influence of any cosmological constant on the properties of the Hubble flow in the local galaxy cluster (LC) and whether the LC can exist in the equilibrium state, at present values of the DE density, where the LC densities of matter consist of the baryonic and dark matter.

Here, we construct Newtonian self-gravitating models with a polytropic equation of state in the presence of DE. In this case, we have a family instead of the single model for each polytropic index $n$. The additional parameter $\beta$ represents the ratio of the density of DE to the matter central density of the configuration. For values of $n=1,1.5,3$, corresponding to the polytropic powers $\gamma=2,5 / 3,4 / 3$, we find the limiting values of $\beta_{\mathrm{c}}$, such that at $\beta>\beta_{\mathrm{c}}$ there are no equilibrium configurations but only an expanding cluster, possibly affected by the Hubble flow.

We derive a virial theorem and analyze the influence of DE on the dynamic stability of the equilibrium models, by using an approximate energetic method. It is shown that DE produces an effect that counteracts the stabilizing influence of the cold dark matter (McLaughlin \& Fuller 1996; Bisnovatyi-Kogan 1998).

\section{Main equations}

We consider a spherically symmetric equilibrium configuration in Newtonian gravity, in the presence of DE, represented by the cosmological constant $\Lambda$. In this case, the gravitational force $F_{\mathrm{g}}$ that a unit mass undergoes in a spherically symmetric body is written as $F_{\mathrm{g}}=-\frac{G m}{r^{2}}+\frac{\Lambda r}{3}$, where $m=m(r)$ is the mass inside the radius $r$. Its connections with the matter density $\rho$ and the equilibrium equation are written respectively as

$\frac{\mathrm{d} m}{\mathrm{~d} r}=4 \pi \rho r^{2}, \quad \frac{1}{\rho} \frac{\mathrm{d} P}{\mathrm{~d} r}=-\frac{G m}{r^{2}}+\frac{\Lambda r}{3}$,

and the DE density $\rho_{v}$ is connected with $\Lambda$ as $\rho_{v}=\frac{\Lambda}{8 \pi G}$. We consider a polytropic equation of state $P=K \rho^{\gamma}$, with $\gamma=1+\frac{1}{n}$. By introducing the non-dimensional variables $\xi$ and $\theta_{n}$ such that

$r=\alpha \xi \quad$ and $\quad \rho=\rho_{0} \theta_{n}{ }^{n}, \quad \alpha^{2}=\frac{(n+1) K}{4 \pi G} \rho_{0}^{\frac{1}{n}-1}$,

we obtain the Lane-Emden equation for polytropic models with DE (see also Balaguera-Antolínez et al. 2007)

$\frac{1}{\xi^{2}} \frac{\mathrm{d}}{\mathrm{d} \xi}\left(\xi^{2} \frac{\mathrm{d} \theta_{n}}{\mathrm{~d} \xi}\right)=-\theta_{n}{ }^{n}+\beta$, 
where $\rho_{0}$ is the matter central density, $\alpha$ is the characteristic radius, and $\beta=\Lambda / 4 \pi G \rho_{0}=2 \rho_{v} / \rho_{0}$ is twice the ratio of the DE density to the central density of the configuration.

\section{The virial theorem}

We first calculate the Newtonian gravitational energy of the configuration in the presence of the cosmological constant. The spherically symmetric Poisson equation for the gravitational potential $\varphi_{*}$ in the presence of $\mathrm{DE}$ is given by

$\Delta \varphi_{*}=\frac{1}{r^{2}} \frac{\mathrm{d}}{\mathrm{d} r}\left(r^{2} \frac{\mathrm{d} \varphi_{*}}{\mathrm{~d} r}\right)=4 \pi G\left(\rho-2 \rho_{v}\right), \quad \varphi_{*}=\varphi+\varphi_{\Lambda}$

The gravitational energy of a spherical body $\varepsilon_{\mathrm{g}}$ is given by

$\varepsilon_{\mathrm{g}}=-G \int_{0}^{M} \frac{m \mathrm{~d} m}{r}, \quad m=4 \pi \int_{0}^{r} \rho r^{2} \mathrm{~d} r, \quad M=m(R)$,

where $R$ is the total radius. For $\varphi_{\Lambda}$ with uniform density $\rho_{v}$ the normalization $\varphi=0$ at $r=\infty$ is impossible. We can then choose $\varphi_{\Lambda}=0$ at $r=0$ as the most convenient normalization. This choice, using Eq. (4), leads to the potential $\varphi_{\Lambda}=-4 \pi G \rho_{v} r^{2} / 3$. Consequently, the energy $\varepsilon_{\Lambda}$, representing the interaction of the matter with $\mathrm{DE}$, is given by

$\varepsilon_{\Lambda}=\int_{0}^{M} \varphi_{\Lambda} \mathrm{d} m=-\frac{4 \pi G \rho_{v}}{3} \int_{0}^{M} r^{2} \mathrm{~d} m$

We find the relations between the gravitational $\varepsilon_{\mathrm{g}}$ and thermal $\varepsilon_{\text {th }}$ energies, and the energy $\varepsilon_{\Lambda}$. For the gravitational energy, we have

$\varepsilon_{\mathrm{g}}=-G \int_{0}^{M} \frac{m \mathrm{~d} m}{r}, \quad m=-\frac{r^{2}}{G}\left(\frac{1}{\rho} \frac{\mathrm{d} P}{\mathrm{~d} r}-\frac{\Lambda r}{3}\right)$,

where $M=m(R)$, and $m$ is written using Eq. (1). For adiabatic systems with a polytropic equation of state, we have $\rho E=n P$ and $I=E+\frac{P}{\rho}=\frac{n+1}{n} E$, where $E$ and $I$ are thermal energy and enthalpy per mass unit. After some transformations, we obtain

$\varepsilon_{\mathrm{g}}=-\frac{3}{n} \varepsilon_{\mathrm{th}}+2 \varepsilon_{\Lambda}, \varepsilon_{\mathrm{tot}}=\frac{3-n}{3} \varepsilon_{\mathrm{g}}+\frac{2 n+3}{3} \varepsilon_{\Lambda}$,

where $\varepsilon_{\text {tot }}=\varepsilon_{\text {th }}+\varepsilon_{\mathrm{g}}+\varepsilon_{\Lambda}, \varepsilon_{\mathrm{th}}=\int_{0}^{M} E \mathrm{~d} m$, while $\varepsilon_{\Lambda}=$ $-\frac{4 \pi G \rho_{v}}{3} \int_{0}^{M} r^{2} \mathrm{~d} m=-\frac{\Lambda}{6} \int_{0}^{M} r^{2} \mathrm{~d} m$ is defined by Eq. (6), and the additive constant in the energy definition of $\varepsilon_{\Lambda}$ is chosen so that $\varepsilon_{\Lambda}=0$ at $\Lambda=0$ or $M=0$. The gravitational energy may also be written as

$\varepsilon_{\mathrm{g}}=-G \int_{0}^{M} \frac{m \mathrm{~d} m}{r}=-\frac{G M^{2}}{2 R}-\frac{G}{2} \int_{0}^{R} \frac{m^{2}}{r^{2}} \mathrm{~d} r$.

We can transform the last integral for polytropic matter by using Eq. (7) and making partial integrations. We have

$$
\begin{aligned}
\frac{G}{2} & \int_{0}^{R} \frac{m^{2}}{r^{2}} \mathrm{~d} r=-\frac{1}{2} \int_{0}^{R} r^{2}\left(\frac{1}{\rho} \frac{\mathrm{d} P}{\mathrm{~d} r}-\frac{\Lambda r}{3}\right) \frac{m}{r^{2}} \mathrm{~d} r \\
= & -\frac{1}{2} \int_{0}^{R} \frac{m}{\rho} \frac{\mathrm{d} P}{\mathrm{~d} r} \mathrm{~d} r+\frac{\Lambda}{6} \int_{0}^{R} m r \mathrm{~d} r=\frac{1}{2} \int_{0}^{M} I \mathrm{~d} m \\
& +\frac{\Lambda}{12} M R^{2}-\frac{\Lambda}{12} \int_{0}^{M} r^{2} \mathrm{~d} m=\frac{n+1}{2 n} \varepsilon_{\mathrm{th}}+\frac{\Lambda}{12} M R^{2}+\frac{\varepsilon_{\Lambda}}{2}
\end{aligned}
$$

Then, by using Eqs. (8) and (10), we obtain from Eq. (9) the relations

$$
\begin{aligned}
\varepsilon_{\mathrm{g}} & =-\frac{3}{5-n} \frac{G M^{2}}{R}-\frac{\Lambda}{2(5-n)} M R^{2}-\frac{2 n+5}{5-n} \varepsilon_{\Lambda}, \\
\varepsilon_{\mathrm{th}} & =\frac{n}{5-n} \frac{G M^{2}}{R}+\frac{n \Lambda}{6(5-n)} M R^{2}+\frac{5 n}{5-n} \varepsilon_{\Lambda} .
\end{aligned}
$$

Finally, by inserting Eq. (12) into (8), we get

$$
\varepsilon_{\mathrm{tot}}=\frac{n-3}{5-n} \frac{G M^{2}}{R}+\frac{(n-3) \Lambda}{6(5-n)} M R^{2}+\frac{2 n}{5-n} \varepsilon_{\Lambda} .
$$

We can calculate $\varepsilon_{\text {tot }}$ for some particular cases. For $n=$ 3 , 1, and 0, we have, respectively, $\varepsilon_{\mathrm{tot}}=3 \varepsilon_{\Lambda}, \quad \varepsilon_{\mathrm{tot}}=-\frac{1}{2} \frac{G M^{2}}{R}-$ $\frac{1}{12} \Lambda M R^{2}+\frac{1}{2} \varepsilon_{\Lambda}$, and $\varepsilon_{\text {tot }}=-\frac{3}{5} \frac{G M^{2}}{R}-\frac{1}{10} \Lambda M R^{2}$. The Lane-Emden model with $n=5$ has an analytical solution with finite mass $M$, finite values of the energies, and an infinite radius $R$, so that must be $(5-n) R \rightarrow$ constant (const.) at $n \rightarrow 5$. In the presence of $\mathrm{DE}$, the finiteness of values of all kinds of energies requires that $(5-n) R \rightarrow$ const. and $\varepsilon_{\Lambda} \rightarrow-\frac{\Lambda}{30} M R^{2}$ at $n \rightarrow 5$. The LaneEmden solution (without DE) at $n=3$ has zero total energy at any given radius and corresponds to a neutral equilibrium. Hence, the knowledge of the total energy of the configuration permits us to identify the boundary between dynamically stable $\left(n<3, \varepsilon_{\text {tot }}<0\right)$ and unstable $\left(n>3, \varepsilon_{\text {tot }}>0\right)$ configurations. In our case, the virial theorem does not permit us to do this, because the value of $\varepsilon_{\Lambda}$ is not properly defined, while the presence of DE in the whole space does not permit us to choose, in a simple way, a universal additive constant of the energy. Therefore, in spite of $\varepsilon_{\text {tot }}=3 \varepsilon_{\Lambda}<0$ at $n=3$ and in accordance with the stability analysis made in Sect. 4 , the polytropic solution at $n=3$ in the presence of DE becomes unstable. Some aspects of the virial theorem in the presence of $\Lambda$ were investigated by Balaguera-Antolínez et al. (2007).

\section{Equilibrium solutions}

The equilibrium mass $M_{n}$ for a generic polytropic configuration that is a solution of the Lane-Emden equation is written as

$M_{n}=4 \pi \int_{0}^{R} \rho r^{2} \mathrm{~d} r=4 \pi\left[\frac{(n+1) K}{4 \pi G}\right]^{3 / 2} \rho_{0}^{\frac{3}{2 n}-\frac{1}{2}} \int_{0}^{\xi_{\text {out }}} \theta_{n}{ }^{n} \xi^{2} \mathrm{~d} \xi$.

Using Eq. (3), the integral on the right side may be calculated by partial integration, giving the relation for the mass of the configuration

$M_{n}=4 \pi \rho_{0} \alpha^{3}\left[-\xi_{\text {out }}^{2}\left(\frac{\mathrm{d} \theta_{n}}{\mathrm{~d} \xi}\right)_{\text {out }}+\frac{\beta \xi_{\text {out }}^{3}}{3}\right]$,

where $\theta_{n}(\xi)$ is not a unique function, but depends on the parameter $\beta$, according to Eq. (3). For the limiting configuration, with $\beta=\beta_{\mathrm{c}}$, we have on the outer boundary $\theta_{n}\left(\xi_{\text {out }}\right)=0,\left.\quad \frac{\mathrm{d} \theta_{n}}{\mathrm{~d} \xi}\right|_{\text {out }}=$ 0 , and the mass $M_{n, \lim }$ of the limiting configuration is written as

$M_{n, \lim }=4 \pi \rho_{0 \mathrm{c}} \alpha^{3} \frac{\beta_{\mathrm{c}} \xi_{\text {out }}^{3}}{3}=\frac{4 \pi}{3} r_{\text {out }}{ }^{3} \beta_{\mathrm{c}} \rho_{0 \mathrm{c}}=\frac{4 \pi}{3} r_{\text {out }}{ }^{3} \bar{\rho}_{\mathrm{c}}$,

such that the limiting value $\beta_{\mathrm{c}}$ is exactly equal to the ratio of the average matter density $\bar{\rho}_{\mathrm{c}}$ of the limiting configuration to its central density $\rho_{0 \mathrm{c}}: \beta_{\mathrm{c}}=\bar{\rho}_{\mathrm{c}} / \rho_{0 \mathrm{c}}$. For the Lane-Emden solution (with $\beta=0$ ), we have $\rho_{0} / \bar{\rho}=3.290,5.99,54.18$ for $n=1,1.5,3$, respectively. We consider the curve $M\left(\rho_{0}\right)$ for a constant DE density $\rho_{v}=\Lambda / 8 \pi G$. In order to plot this curve 


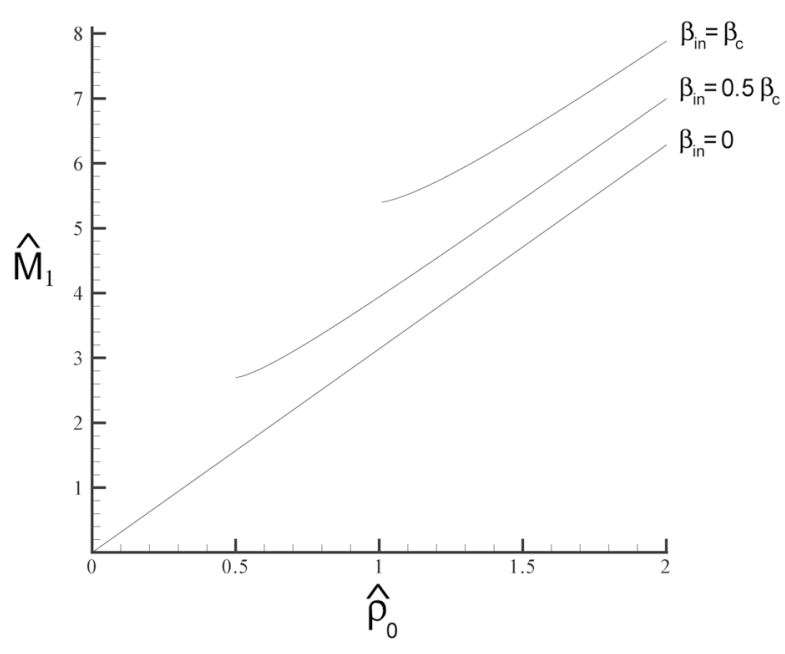

Fig. 1. Non-dimensional mass $\hat{M}_{1}$ of the equilibrium polytropic configurations at $n=1$ as a function of the non-dimensional central density $\hat{\rho}_{0}$, for different values of $\beta_{\text {in }}$. The cosmological constant $\Lambda$ is the same along each curve. The curves at $\beta_{\text {in }} \neq 0$ are limited by the configuration with $\beta=\beta_{\mathrm{c}}$.

in the non-dimensional form, we introduce an arbitrary scaling constant $\rho_{\mathrm{ch}}$ and write the expression for the mass in the form $M_{n}=4 \pi\left[\frac{(n+1) K}{4 \pi G}\right]^{3 / 2} \rho_{\mathrm{ch}}^{\frac{3}{2 n}-\frac{1}{2}} \hat{M}_{n}$, with

$\hat{M}_{n}=\hat{\rho}_{0}^{\frac{3}{2 n}-\frac{1}{2}}\left[\frac{\beta \xi_{\text {out }}^{3}}{3}-\xi_{\text {out }}^{2}\left(\frac{\mathrm{d} \theta_{n}}{\mathrm{~d} \xi}\right)_{\text {out }}\right]$,

where $\hat{\rho}_{0}=\rho_{0} / \rho_{\text {ch }}$ is the non-dimensional central density. We also introduced the non-dimensional mass $\hat{M}_{n}$.

At $n=1$, Eq. (3) is linear and has an analytic solution (Chandrasekhar 1939). The solution satisfying the boundary conditions at the center, $\theta_{1}(0)=1, \theta_{1}{ }^{\prime}(0)=0$, is written as $\theta_{1}=(1-\beta) \frac{\sin \xi}{\xi}+\beta$. The radius of the configuration is determined by the transcendental equation $(1-\beta) \frac{\sin \xi_{\text {out }}}{\xi_{\text {out }}}+\beta=0$. This equation only has real solutions at $\beta<\beta_{\mathrm{c}}$, such that at the outer boundary not only does $\theta_{1}=0$, but also $\theta_{1}{ }^{\prime}=0$ for $\beta=\beta_{\mathrm{c}}$. We have $\theta_{1}{ }^{\prime}=(1-\beta)\left(\frac{\cos \xi}{\xi}-\frac{\sin \xi}{\xi^{2}}\right)$. Therefore, the parameters $\beta_{\mathrm{c}}$ and $\xi_{\text {out,c }}$ of the limiting equilibrium solution in the presence of DE are determined by the algebraic equations $\left(1-\beta_{\mathrm{c}}\right) \frac{\sin \xi_{\text {out }, \mathrm{c}}}{\xi_{\text {out. }}}+\beta_{\mathrm{c}}=0$ and $\tan \xi_{\text {out }, \mathrm{c}}=\xi_{\text {out }, \mathrm{c}}$, where $\pi<\xi_{\text {out }, \mathrm{c}}<3 \pi / 2$. At large $\xi$, the solutions asymptotically approach the horizontal line $\theta_{1}=\beta$. Our numerical analysis indicates that $\xi_{\text {out }}=\pi, 3.490,4.493$, for $\beta=0, \beta=0.5 \beta_{\mathrm{c}}=0.089$, and $\beta=\beta_{\mathrm{c}}=0.178$, respectively. We plot the non-dimensional curve $\hat{M}_{n}\left(\hat{\rho}_{0}\right)$, at constant $\rho_{v}=\beta \rho_{0} / 2$. We construct the curve starting from the model with $\hat{\rho}_{0}=1$ at different $\beta$, and then following the sequence by varying the central density $\hat{\rho}_{0}$ assuming that $\beta \propto 1 / \hat{\rho}_{0}$, at $\beta \leq \beta_{\mathrm{c}}$. For $n=1$, we have

$\hat{M}_{1}=\hat{\rho}_{0}\left[(1-\beta)\left(\sin \xi_{\text {out }}-\xi_{\text {out }} \cos \xi_{\text {out }}\right)+\frac{\beta \xi_{\text {out }}^{3}}{3}\right]$,

where $\hat{\rho}_{0} \beta=\beta_{\text {in }}=$ const. The behavior of $\left.\hat{M}_{1}\left(\hat{\rho}_{0}\right)\right|_{\Lambda}$ is given in Fig. 1 for $\beta_{\text {in }}=0, \beta_{\text {in }}=0.5 \beta_{\mathrm{c}}$, and $\beta_{\text {in }}=\beta_{\mathrm{c}}$, for which $\hat{M}_{1}=\pi, 3.941,5.397$ at $\hat{\rho}_{0}=1$. We note that for $\beta_{\text {in }}=\beta_{\text {c }}$ there are equilibrium models only for $\hat{\rho}_{0}>1$.

At $n=3$, the mass of the configuration is given by $M_{3}=$ $4 \pi\left[\frac{K}{\pi G}\right]^{3 / 2} \hat{M}_{3}$, where $\hat{M}_{3}$ is derived by Eq. (17). The LaneEmden model $(\beta=0)$ has a unique value of the mass that is

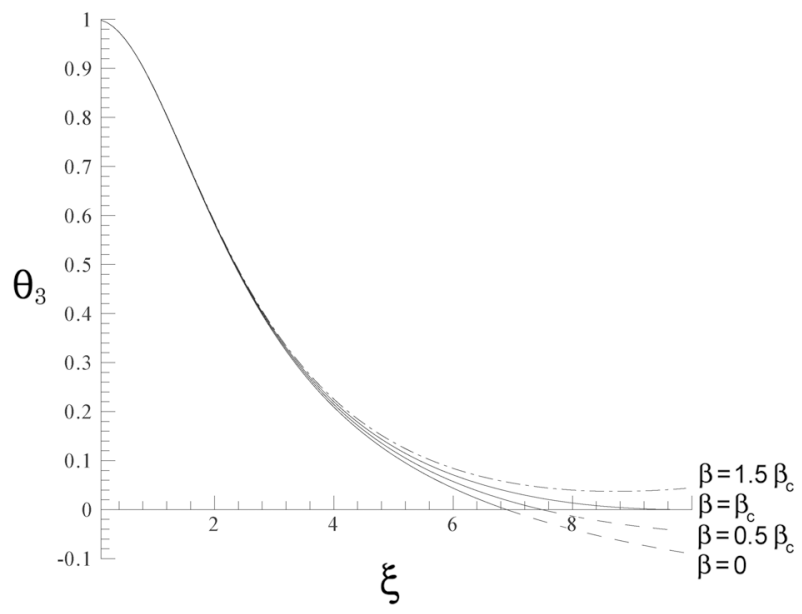

Fig. 2. The density distribution for configurations at $n=3$ with $\beta=$ $0, \beta=0.5 \beta_{\mathrm{c}}$, and $\beta=\beta_{\mathrm{c}}$. The curves are marked with the values of $\beta$. The non-physical solution at $\beta=1.5 \beta_{\mathrm{c}}$, which does not have an outer boundary, is given by the dash-dot line. The non-physical parts of the solutions at $\beta \leq \beta_{\mathrm{c}}$, behind the outer boundary, are given by the dash lines. The solutions asymptotically approach, at large $\xi$, the horizontal line $\theta_{3}=\beta^{1 / 3}$.

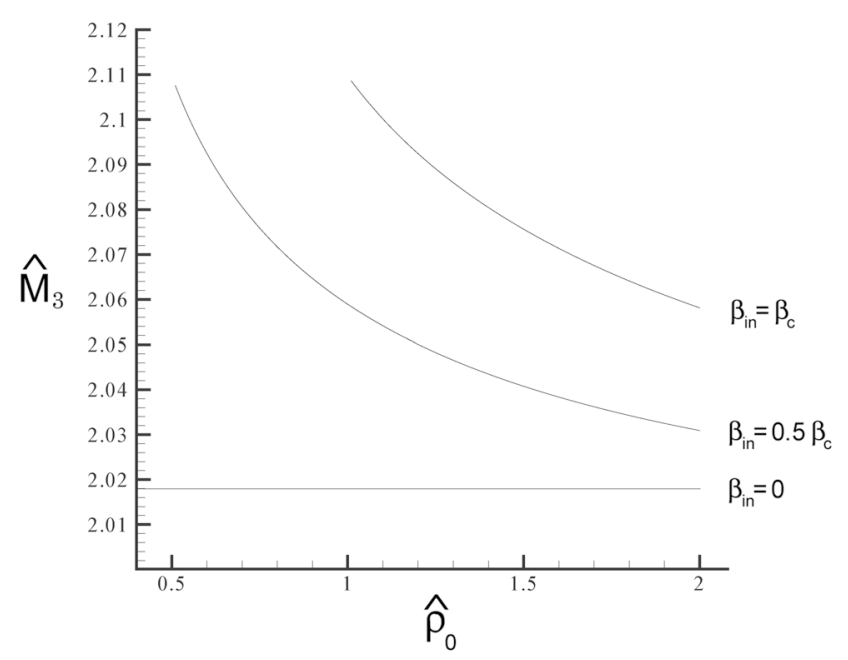

Fig. 3. Same as in Fig. 1, for $n=3$.

independent of the density (equilibrium configuration with neutral dynamical stability). At $\beta \neq 0$, the model's dependence on the density appears because the function $\theta_{3}$ is different for different values of $\beta$ and, along the curve $\left.\hat{M}_{3}\left(\hat{\rho}_{0}\right)\right|_{\Lambda}$, the value of $\beta$ is inversely proportional to $\hat{\rho}_{0}$.

The density distribution for equilibrium configurations with $\beta=0, \beta=0.5 \beta_{\mathrm{c}}$, and $\beta=\beta_{\mathrm{c}}$ is shown in Fig. 2. At large $\xi$, these solutions asymptotically approach the horizontal line $\theta_{3}=\beta^{1 / 3}$, with damping oscillations around this value. The numerical solution of the equilibrium equation gives $\xi_{\text {out }}=$ $6.897,7.489,9.889$, for $\beta=0, \beta=0.5 \beta_{\mathrm{c}}=0.003$, and $\beta=\beta_{\mathrm{c}}=0.006$, respectively. In Fig. 3, we show the behavior of $\left.\hat{M}_{3}\left(\hat{\rho}_{0}\right)\right|_{\Lambda}$, for different values of $\beta_{\text {in }}=0, \beta_{\text {in }}=0.5 \beta_{\mathrm{c}}$, and $\beta_{\text {in }}=\beta_{\mathrm{c}}$, for which $\hat{M}_{3}=2.018,2.060,2.109$, at $\hat{\rho}_{0}=1$, respectively.

The behavior of $\left.\hat{M}_{3}\left(\hat{\rho}_{0}\right)\right|_{\Lambda}$ in Fig. 3, showing a decreasing mass with increasing central density, corresponds, for an adiabatic index equal to the polytropic one, to dynamically unstable 
configurations, according to the static criterion of stability (Zel'dovich 1963). When the vacuum influence is small, it is possible to investigate the stability of the adiabatic configuration by the approximate energetic method (Zeldovich \& Novikov 1966; Bisnovatyi-Kogan 2001). For $n=3$, at $\rho_{0} \gg \rho_{v}$, the density in the configuration is distributed according to the LaneEmden solution $\rho=\rho_{0} \theta_{3}^{3}(\xi)$. In this case, we may investigate the stability to homologous perturbations by changing only the central density at fixed density distribution, given by the function $\theta_{3}$.

We consider configurations close to the polytropic (adiabatic) equilibrium solution at $n=3$ (and $\beta=0$ ), where the turning point of stability is expected. In this case, the presence of DE does not affect significantly the gravitational equilibrium, thus the unperturbed polytropic solution at $n=3$ can be used to calculate the gravitational energy $\varepsilon_{\mathrm{g}}$. The energy $\varepsilon_{*}$ will then be given by

$\varepsilon_{*}=\varepsilon_{\mathrm{g}}+\varepsilon_{\Lambda}=-G \int_{0}^{M} \frac{m \mathrm{~d} m}{r}-\frac{4 \pi G \rho_{v}}{3} \int_{0}^{M} r^{2} \mathrm{~d} m$,

thus $\varepsilon_{*}=-\frac{3}{2} \frac{G M^{2}}{R}-\frac{\Lambda}{6} \int_{0}^{M} r^{2} \mathrm{~d} m$, where, taking into account the non-dimensional variables in Eq. (2), the energy $\varepsilon_{\Lambda}$ can be written as

$\varepsilon_{\Lambda}=-\frac{\Lambda}{6} \int_{0}^{M} r^{2} \mathrm{~d} m=-\frac{2}{3} \pi \rho_{0} \alpha^{5} \Lambda \int_{0}^{\xi_{\text {out }}} \theta_{3}^{3} \xi^{4} \mathrm{~d} \xi$,

where $\int_{0}^{\xi_{\text {out }}} \theta_{3}{ }^{3} \xi^{4} \mathrm{~d} \xi=10.85$ (see Bisnovatyi-Kogan 2001). In the analysis of the dynamical stability, we consider the total energy $\varepsilon$ of the configuration, taking into account a small correction $\varepsilon_{\mathrm{GR}}$ due to general relativistic effects. We have

$$
\begin{aligned}
\varepsilon= & \varepsilon_{\mathrm{th}}+\varepsilon_{\mathrm{g}}+\varepsilon_{\Lambda}+\varepsilon_{\mathrm{GR}}=\int_{0}^{M} E \mathrm{~d} m-0.639 G M^{5 / 3} \rho_{0}^{1 / 3} \\
& -0.104 \Lambda M^{5 / 3} \rho_{0}^{-2 / 3}-0.918 \frac{G^{2} M^{7 / 3}}{c^{2}} \rho_{0}^{2 / 3},
\end{aligned}
$$

where we used the relations for the polytropic configuration with $n=3, \xi_{\text {out }}=6.897$, and $R=\alpha \xi_{\text {out }}=\frac{M^{1 / 3} \rho_{0}^{-1 / 3}}{0.426}$. The equilibrium configuration is determined by the zero of the first derivative of $\varepsilon$ over $\rho_{0}$, at constant entropy $S$ and mass $M$, while the stability of the configuration is analyzed in terms of the sign of the second derivative: if positive, the configuration is dynamically stable, if negative, the configuration is unstable. It is more convenient to take derivatives over $\rho_{0}^{1 / 3}$ than over $\rho_{0}$. Thus

$$
\begin{aligned}
\frac{\partial \varepsilon}{\partial \rho_{0}^{1 / 3}}= & 3 \rho_{0}^{-4 / 3} \int_{0}^{M} P \frac{\mathrm{d} m}{\phi(m / M)}-0.639 G M^{5 / 3} \\
& +0.208 \Lambda M^{5 / 3} \rho_{0}^{-1}-1.84 \frac{G^{2} M^{7 / 3}}{c^{2}} \rho_{0}^{1 / 3}=0
\end{aligned}
$$

for the equilibrium, and the sign of the second derivative

$$
\frac{9}{\rho_{0}^{5 / 3}} \int_{0}^{M}\left(\gamma-\frac{4}{3}\right) \frac{P \mathrm{~d} m}{\phi(m / M)}-0.623 \Lambda M^{5 / 3} \rho_{0}^{-4 / 3}-1.84 \frac{G^{2} M^{7 / 3}}{c^{2}}
$$

for the analysis of the dynamical stability, where $\gamma=\left(\frac{\rho}{P} \frac{\partial P}{\partial \rho}\right)_{S}$ and $\rho=\rho_{0} \phi\left(\frac{m}{M}\right)$ are the adiabatic index $\gamma$ at constant entropy $S$ and the non-dimensional function $\phi$, which both remain constant during homologous perturbations, respectively. It follows from Eq. (23) that DE input in the stability of the configuration is

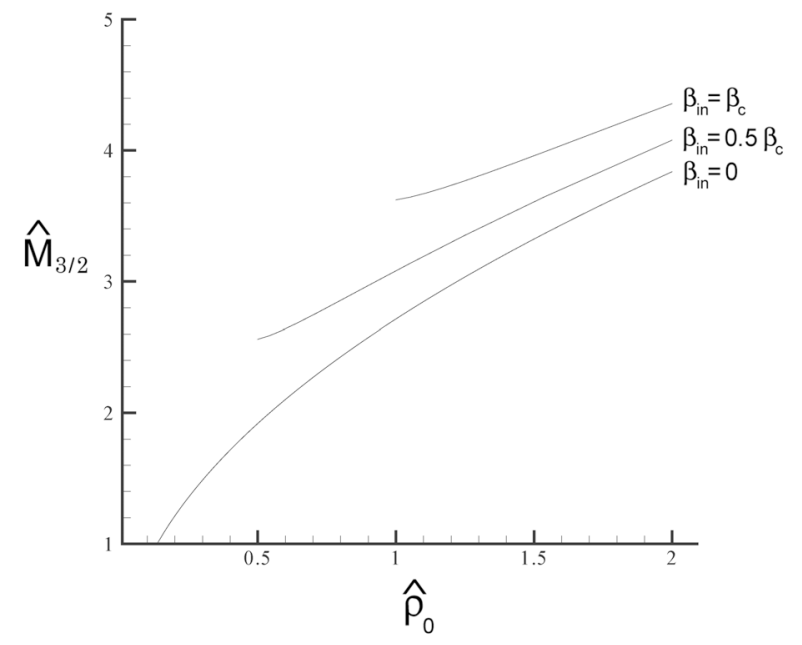

Fig. 4. Same as in Fig. 1, for $n=3 / 2$.

negative, as in the general relativistic correction (Chandrasekhar 1964; Merafina \& Ruffini 1989). Therefore, an adiabatic star with a polytropic index of $4 / 3$ becomes unstable in the presence of DE. The dynamic stability of pure polytropic models was also investigated by Balaguera-Antolínez et al. (2006, 2007), by using a static criterion of stability. Our criterion is valid for any equation of state $P(\rho, T)$.

At $n=1.5$, the mass of the configuration is written as $M_{3 / 2}=4 \pi\left[\frac{5 K}{8 \pi G}\right]^{3 / 2} \rho_{\mathrm{ch}}^{1 / 2} \hat{M}_{3 / 2}$, where $\hat{M}_{3 / 2}$ is derived by Eq. (17). At large $\xi$, these solutions asymptotically approach the horizontal line $\theta_{3 / 2}=\beta^{2 / 3}$. The numerical solution gives $\xi_{\text {out }}=$ $3.654,3.984,5.086$, for $\beta=0, \beta=0.5 \beta_{\mathrm{c}}=0.041, \beta=$ $\beta_{\mathrm{c}}=0.082$, respectively. In Fig. 4 , we show the behavior of $\left.\hat{M}_{3 / 2}\left(\hat{\rho}_{0}\right)\right|_{\Lambda}$, for different values of $\beta_{\text {in }}=0, \beta_{\text {in }}=0.5 \beta_{\mathrm{c}}$, and $\beta_{\text {in }}=\beta_{\mathrm{c}}$, for which $\hat{M}_{3 / 2}=2.714,3.081,3.622$, at $\hat{\rho}_{0}=1$, respectively.

\section{Discussion}

The question about the importance of DE to the dynamics of the Local Cluster (LC) was raised by Chernin (2008). For presently accepted values of the DE density $\rho_{v}=(0.72 \pm 0.03) \times$ $10^{-29} \mathrm{~g} / \mathrm{cm}^{3}$, the mass of the Local Group, including its dark matter input, is between $M_{\mathrm{LC}} \sim 3.5 \times 10^{12} M_{\odot}$, according to Chernin et al. (2009), and $M_{\mathrm{LC}} \sim 1.3 \times 10^{12} M_{\odot}$, according to Karachentsev et al. (2006). The radius $R_{\mathrm{LC}}$ of the LC is even more poorly known. It can be estimated by measuring the velocity dispersion $v_{\mathrm{t}}$ of galaxies in the LC and by the application of the virial theorem, such that $R_{\mathrm{LC}} \sim\left(G M_{\mathrm{LC}} / v_{\mathrm{t}}^{2}\right)$. The estimated velocity dispersion of galaxies in the $\mathrm{LC}$, which has been found to equal $v_{\mathrm{t}}=63 \mathrm{~km} \mathrm{~s}^{-1}$, is very close to the value of the local Hubble constant $H=68 \mathrm{~km} \mathrm{~s}^{-1} \mathrm{Mpc}^{-1}$ (Karachentsev et al. 2006). The similarity between these values indicates the great difficulties in dividing the measured velocities between regular and chaotic components. The radius of the LC may be estimated to be $R_{\mathrm{LC}}=\left(G M_{\mathrm{LC}} / v_{\mathrm{t}}^{2}\right)$, and to have values between $1.5 \mathrm{Mpc}$ and $4 \mathrm{Mpc}$ and a very large error box that we cannot estimate properly. Chernin et al. (2009) identifies the radius $R_{\mathrm{LC}}$ with the radius $R_{v}$ of the zero-gravity force, which is identical to the one corresponding to our critical model with $\beta=\beta_{\mathrm{c}}$, in which the average matter density is equal to $2 \rho_{v}: 1.2<M_{\mathrm{LC}}<3.7 \times 10^{12} M_{\odot}$ and $1.1<R_{v}<1.6 \mathrm{Mpc}$. All these estimations show the importance of the presently accepted value of DE density on the 
M. Merafina et al.: A brief analysis of self-gravitating polytropic models with a non-zero cosmological constant $(R N)$

structure and dynamics of the outer parts of LC, and its vicinity. Polytropic solutions with DE are inappropriate for describing the LC, but may be more applicable to rich galactic clusters.

Acknowledgements. The work of GSBK and SOT was partially supported by RFBR grant 11-02-00602, the Presidium RAN program P20 and RF President Grant NSh-3458.2010.2.

\section{References}

Balaguera-Antolínez, A., Mota, D. F., \& Nowakowski, M. 2006, Class. Quant. Grav., 23, 4497

Balaguera-Antolínez, A., Mota, D. F., \& Nowakowski, M. 2007, MNRAS, 382 , 621

Bisnovatyi-Kogan, G. S. 1998, ApJ, 497, 559
Bisnovatyi-Kogan, G. S. 2001, Stellar Physics, II. Stellar Structure and Stability (Heidelberg: Springer)

Chandrasekhar, S. 1939, An Introduction to the Study of Stellar Structure (Chicago: Univ. Chicago Press)

Chandrasekhar, S. 1964, ApJ, 140, 417

Chernin, A. D. 2008, Phys. Usp., 51, 267

Chernin, A. D., Teerikorpi, P., Valtonen, M. J., et al. 2009, ApJL, submitted [arXiv:astro-ph0902.3871v1]

Karachentsev, I. D., Dolphin, A., Tully, R. B., et al. 2006, AJ, 131, 1361

McLaughlin, G., \& Fuller, G. 1996, ApJ, 456, 71

Merafina, M., \& Ruffini, R. 1989, A\&A, 221, 4

Perlmutter, S., Aldering, G., Goldhaber, G., et al. 1999, ApJ, 517, 565

Riess, A. G., Filippenko, A. V., Challis, P., et al. 1998, AJ, 116, 1009

Spergel, D. N., Verde, L., Peiris, H. V., et al. 2003, ApJS, 148, 175

Zel'dovich, Ya. B. 1963, Voprosy Kosmogonii, 9, 157

Zel'dovich, Ya. B., \& Novikov, I. D. 1966, Phys. Usp., 8, 522 